\title{
Congenital colonic stenosis diagnosed in adulthood
}

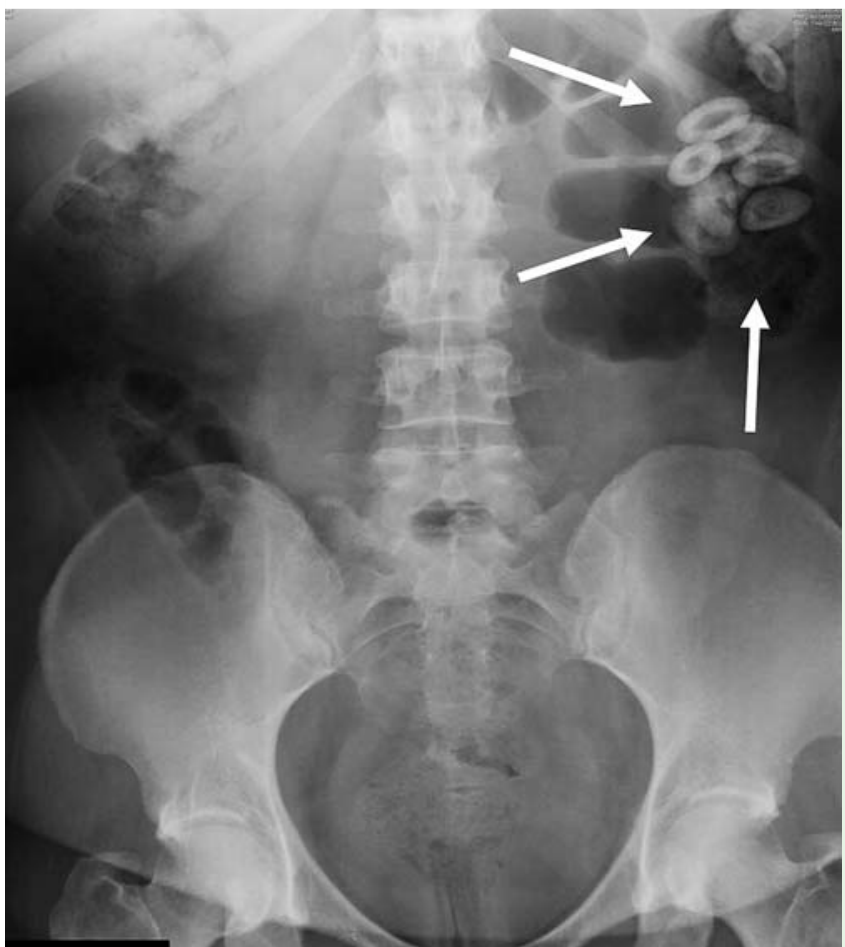

Fig. 1 Abdominal radiograph showing calcified fecalomas at the splenic flexure (arrows).

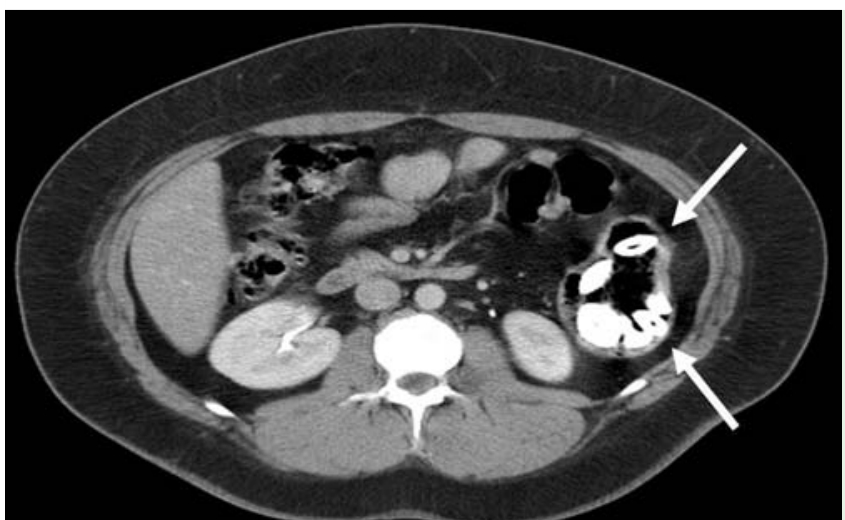

Fig. 2 Axial computed tomography (CT) scan showing calcified fecalomas at the splenic flexure (arrows).

A 32-year-old immigrant woman presented with abdominal pains, nausea, and vomiting. She had not been able to expel gas or feces for 5 days. She had had constipation since birth and had experienced many episodes similar to the current bout. The patient had been hospitalized during infancy in her country, but the cause for her complaint had not been identified. She regularly took laxatives as required. On examination, her abdomen was bloated and tympanic with diffuse tenderness. The rest of the examination and laboratory tests were normal. An ab- dominal radiograph ( $\bullet$ Fig. 1 ) and computed tomography scan ( $\bullet$ Fig. 2) showed a dilated ascending and transverse colon, and oval lesions with a radiopaque peripheral ring in the upper left quadrant. Colonoscopy ( $\bullet$ Fig. 3 ) revealed stenosis of the splenic flexure, which was covered by normal mucosa. The patient underwent surgical resection. Histological studies revealed a normal colon with a $2-\mathrm{cm}$ concentric stenosis of the wall and hypertrophy of the muscular layer. There were no inflammatory, ischemic, or neoplastic changes, or changes in the nerve plexuses,

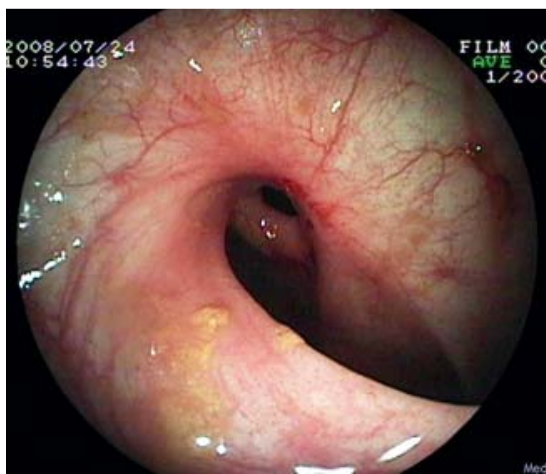

Fig. 3 Colonoscopy showing benign stenosis of the splenic flexure.

but calcified fecalomas were seen proximally. At 12 months of follow-up, the patient remains asymptomatic with daily bowel movements that do not require taking laxatives.

Congenital stenosis can affect any section of the colon, although it most often occurs in the ascending and transverse segments. Its clinical manifestations are usually chronic constipation and obstructive episodes $[1,2]$, and it generally is treated by surgical resection within 24 months of birth [3]. In our case, the onset of the symptoms in the neonatal period, the absence of a clinical or pathoanatomical history of inflammatory or ischemic abdominal processes, and the presence of calcified fecalomas suggested a diagnosis of congenital colonic stenosis that had not been identified during infancy. To our knowledge, it is the first report of congenital stenosis diagnosed in adulthood.

\section{Competing interests: None}

Endoscopy_UCTN_Code_CCL_1AD_2AJ

\section{J. S. Baudet ${ }^{1}$, I. Redondo ${ }^{1}$, X. Arguiña-} rena $^{1}$, V. Castro ${ }^{2}$, M. Alonso ${ }^{3}$

Digestive System Research, Hospital Universitario Ntra, Sra. de Candelaria, Santa Cruz de Tenerife, Canary Islands, Spain

2 Pathology Service, Hospital Universitario Ntra, Santa Cruz de Tenerife, Canary Islands, Spain

3 Surgical Service, Hospital Universitario Ntra, Santa Cruz de Tenerife, Canary Islands, Spain 


\section{References}

1 García Vázquez A, Cano Novillo I, Portela Casalod $E$ et al. Congenital colonia stenosis. An Esp Pediatr 2002; 56: 258-260

2 Winters WD, Weinberger E, Hatch EI. Atresia of the colon in neonates: Radiographic findings. Am J Rheumatol 1992; 159: $1273-$ 1276

3 Sax EJ. Congenital colonic stenosis. Am J Rheumatol 1991; 156: 1315-1317
Bibliography

DOI 10.1055/s-0029-1243941

Endoscopy 2010; 42: E188 -E189

(c) Georg Thieme Verlag KG Stuttgart · New York . ISSN 0013-726X
Corresponding author

J. S. Baudet, MD

Digestive System Research Hospital Universitario Ntra

Sra. de Candelaria

38010 Santa Cruz de Tenerife

Canary Islands

Spain

baudetjs@yahoo.es 